\title{
ANALYTIC SEPARABLE DICTIONARY LEARNING BASED ON OBLIQUE MANIFOLD
}

\author{
Fengzhen Zhang ${ }^{a, b}$, Yigang Cen ${ }^{a, b, *}$, Ruizhen Zhao ${ }^{a, b}$, Hengyou Wang ${ }^{a, b, c}$, Yi \\ $\mathrm{Cen}^{d}$, LiHong Cui ${ }^{e}$, ShaoHai $\mathrm{Hu}^{a, b}$ \\ ${ }^{a}$ Institute of Information Science, Beijing Jiaotong University, Beijing, China; \\ ${ }^{b}$ Key Laboratory of Advanced Information Science and Network Technology of Beijing, Beijing, \\ China; \\ ${ }^{c}$ School of Science, Beijing University of Civil Engineering and Architecture, Beijing, China; \\ ${ }^{d}$ School of Information Engineering, Minzu University of China, Beijing, China; \\ ${ }^{e}$ Department of Mathematics and Computer Science, Beijing University of Chemical Technology, \\ Beijing, China
}

\begin{abstract}
Sparse representation based on dictionary has gained increasing interest due to its extensive applications. Because of the disadvantages of computational complexity of traditional dictionary learning, we propose an algorithm of analytic separable dictionary learning. Considering the differences of sparse coefficient matrix and dictionary, we divide our algorithm into two phases: 2D sparse coding and dictionary optimization. Then an alternative iteration method is used between these two phases. The algorithm of 2D-OMP (2-dimensional Orthogonal Matching Pursuit) is used in the first phase because of its low complexity. In the second phase, we create a continuous function of the optimization problem, and solve it by the conjugate gradient method on oblique manifold. By employing the separable structure of the optimized dictionary, a competitive result is achieved in our experiments for image de-noising.
\end{abstract}

Keywords:

Dictionary learning, oblique manifold, sparse representation, 2D-OMP

\section{Introduction}

Recently, sparse representation has received many attentions for its extensive applications to face recognition [1] and image processing, such as image denoising [2], image super resolution reconstruction [3-5], etc. The model supposes 
that a signal can be represented as a sparse linear combination of a few columns of a dictionary, i.e., suppose that $\mathbf{D} \in \mathbb{R}^{m^{2} \times n^{2}}$ is a dictionary with $m \ll n$, a signal $\mathbf{y} \in \mathbb{R}^{m^{2}}$ can be expressed as $\mathbf{y} \approx \mathbf{D} \mathbf{x}$, such that $\|\mathbf{y}-\mathbf{D} \mathbf{x}\|_{p} \leqslant \epsilon$. Here the vector $\mathbf{x} \in \mathbb{R}^{n^{2}}$ is a sparse vector, i.e., $\|\mathbf{x}\|_{0}=k \ll m^{2}$, which means that most of its entries are zeros or have small magnitudes [6-10].

The properties of dictionary $\mathbf{D}$ decide the sparsity of $\mathbf{y}$. The constraints on the dictionary include [6, 7]:

(1)The columns of dictionary have unit Euclidean norm, i.e., $\left\|\mathbf{D}_{i}\right\|_{2}=1$ for $i=$ $1,2, \cdots, n^{2}$.

(2)The dictionary has a full rank, i.e., $\operatorname{rank}(\mathbf{D})=m^{2}$.

(3)The dictionary does not have linearly dependent columns, i.e., $\mathbf{D}_{i} \neq \pm \mathbf{D}_{j}$ for $i \neq j$.

Therefore, it is crucial to find a dictionary so that the interested signal can be represented as accurate as possible with a coefficient vector $\mathbf{x}$ that is as sparse as possible $[9,10]$. There are two different types of dictionaries: analytic dictionaries and learned dictionaries [11]. In many cases, the analytic dictionaries can lead to simple and rapid algorithms for the problem of sparse representation, such as Wavelets [12], Curvelets, and Fourier transform matrix. But for some special signals, e.g., the natural facial images, the analytic dictionaries cannot lead to a perfect result, because the structure of signals is so complicated that a simple dictionary cannot capture the most salient features of them. Thus, algorithms of dictionary construction were proposed based on learning samples [10, 13-15]. The typical dictionary learning problem is

$$
\underset{\mathbf{D}, \mathbf{X}}{\operatorname{argmin}}\|\mathbf{X}\|_{0} \quad \text { s.t. }\|\mathbf{D X}-\mathbf{Y}\|_{F}^{2} \leqslant \epsilon
$$

Here, $\|*\|_{0}$ denotes the $\ell_{0}$ norm, which counts the nonzero entries of a matrix. $\mathbf{Y}=\left[\mathbf{Y}_{1}, \mathbf{Y}_{2}, \cdots, \mathbf{Y}_{N}\right] \in \mathbb{R}^{m^{2} \times N}$ is the matrix containing $N$ learning samples, and the matrix $\mathbf{X}=\left[\mathbf{X}_{1}, \mathbf{X}_{2}, \cdots, \mathbf{X}_{N}\right] \in \mathbb{R}^{n^{2} \times N}$ contains the corresponding sparse coefficients.

The most popular dictionary learning algorithm is the K-SVD, which is an alternative iteration method $[10,15,16]$. Recently, because of the classification inability of K-SVD, discriminative K-SVD was proposed [17]. In [11], a Fisher Discriminative K-SVD (FD-KSVD) was proposed, which not only employed the Fisher discrimination criterion to obtain discriminative coding coefficients, but also introduced a linear predictive classifier. In [6], they presented an analysis sparse model and projected the analysis dictionary to the oblique manifold due to the constraints of the dictionary. In this algorithm, the dictionary is updated as a 
whole part, which is different with the previous algorithms that updated the atoms of dictionary one by one. However, these algorithms are used for 1D vectors. When we deal with 2D signals, such as natural images, we need to convert images to $1 \mathrm{D}$ vectors. This kind of process not only breaks the potential correlations within the images, but is also restricted due to limitations in memory and computational ability $[6,7,18]$. Thus, separable learning schemes were proposed, which tackled this problem by enforcing additional structure on the learned dictionary, as follows:

$$
\begin{array}{cl}
\underset{\mathbf{A},\left\{\mathbf{X}_{j}\right\}_{j=1}^{N}, \mathbf{B}}{\arg \min } & \frac{1}{N} \sum_{j=1}^{N}\left\|\mathbf{X}_{j}\right\|_{0} \\
\text { s.t. } & \mathbf{A X}_{j} \mathbf{B}^{T}=\mathbf{S}_{j}, \mathbf{X}_{j} \in \mathbb{R}^{n_{\mathbf{A}} \times n_{\mathbf{B}}}, \\
& \operatorname{ddiag}\left(\mathbf{A}^{T} \mathbf{A}\right)=\mathbf{I}_{n_{\mathbf{A}}}, \operatorname{rank}(\mathbf{A})=m_{\mathbf{A}}, \\
& \operatorname{ddiag}\left(\mathbf{B}^{T} \mathbf{B}\right)=\mathbf{I}_{n_{\mathbf{B}}}, \operatorname{rank}(\mathbf{B})=m_{\mathbf{B}} .
\end{array}
$$

Here, $\operatorname{ddiag}(*)$ denotes a diagonal matrix consisting of the main diagonal elements of $* .\left\{\mathbf{S}_{j}\right\}_{j=1}^{N}$ are the learning samples and $\mathbf{S}_{j} \in \mathbb{R}^{m_{\mathbf{A}} \times m_{\mathbf{B}}}, \forall j=1, \cdots, N$. The sizes of $\mathbf{A} \in \mathbb{R}^{m_{\mathbf{A}} \times n_{\mathbf{A}}}$ and $\mathbf{B} \in \mathbb{R}^{m_{\mathbf{B}} \times n_{\mathbf{B}}}$ may be different. In this paper, we let $m_{\mathbf{A}}=m_{\mathbf{B}}=m, n_{\mathbf{A}}=n_{\mathbf{B}}=n$ for simplicity.

In [16], 2D synthesis sparse model was proposed, which made full use of the local correlations within natural images. In this model, the dictionary $\mathbf{D} \in$ $\mathbb{R}^{m^{2} \times n^{2}}$ has a separable structure, which can be given by the Kronecker product of two smaller dictionaries $\mathbf{A} \in \mathbb{R}^{m \times n}$ and $\mathbf{B} \in \mathbb{R}^{m \times n}$, formulated as $\mathbf{D}=\mathbf{B} \otimes \mathbf{A}$. Then, it can be seen as a combination of twice K-SVD along the horizontal and vertical directions, respectively. In [7], based on the same assumption of the structure of $\mathbf{D}$, they used a continuous function to measure the sparsity and optimize the problem through the conjugate gradient algorithm. With the constraints on $\mathbf{A}$ and $\mathbf{B}$, they restricted these two dictionaries to be elements of the oblique manifold, i.e., $O B(m, n):=\left\{\mathbf{Q} \in \mathbb{R}^{m \times n} \mid \operatorname{rank}(\mathbf{Q})=m, \operatorname{ddiag}\left(\mathbf{Q}^{T} \mathbf{Q}\right)=\mathbf{I}_{n}\right\}$. However, they ignored the influence of dictionary rank condition for the sparse representation. Also, a same step size was used for updating $\mathbf{A}, \mathbf{B}$, and $\left\{\mathbf{X}_{j}\right\}_{j=1}^{N}$.

In this paper, we propose an algorithm for analytic separable dictionary learning. Inspired by the K-SVD, we optimize the sparse coefficients and the dictionaries in two phases. In the first phase, the sparse coefficients $\left\{\mathbf{X}_{j}\right\}_{j=1}^{N}$ are updated by the 2D-OMP(2-dimensional Orthogonal Matching Pursuit). In the second phase, the dictionaries are optimized with the given $\left\{\mathbf{X}_{j}\right\}_{j=1}^{N}$. This optimization procedure alternated between these two phases until the stop criterion is satisfied. The main contributions are as follows: 
(1) Different with [7] that updates $\left\{\mathbf{X}_{j}\right\}_{j=1}^{N}, \mathbf{A}$ and $\mathbf{B}$ in a same step size, they are updated respectively in our proposed algorithm. 2D-OMP is used to update $\left\{\mathbf{X}_{j}\right\}_{j=1}^{N}$, which not only ensures the sparsity of $\left\{\mathbf{X}_{j}\right\}_{j=1}^{N}$, but also speeds up the convergence of the objective function. Moreover, $\mathbf{A}$ and $\mathbf{B}$ are projected onto the oblique manifold and updated together, which uses the matrix multiplication instead of the SVD decomposition used in the traditional K-SVD. This will save the computational cost for the dictionary optimization, especially when the dictionary dimension is high.

(2) To emphasize the constraints of full rank and incoherence, two log-barrier functions are added to the objective function such that a continuous differentiable objective function can be obtained, which ensures that the conjugate gradient method can be applied to optimize the dictionary. Also, full rank constraint will improve the robustness of the optimized dictionary.

(3) Finally, an analytic separable dictionary learning algorithm is proposed, which not only reduces the computational cost of the dictionary optimization, but also optimizes the dictionary quickly even in the high-dimensional situation.

The remainder of this paper is organized as follows. In Section II, we describe our proposed approach in details. The results are presented in Section III. Section IV concludes this paper.

\section{Learning Schemes}

To solve the optimization problem (2), we divide it into two phases. The first phase calculates the sparse matrices $\left\{\mathbf{X}_{j}\right\}_{j=1}^{N}$ with the given dictionaries, which is called as the 2D sparse coding. In the second phase, we add the constraints of dictionaries through coefficients to the objective function. Due to the constraints of the dictionary and the continuous differentiable objective function, the dictionaries $\mathbf{A}$ and $\mathbf{B}$ are projected to the oblique manifold, and the geometric conjugate gradient method is employed to solve this function. By projecting the dictionaries to the oblique manifold, they can be updated as a whole part, which avoids updating the atoms of dictionary one by one such that the updating speed can be improved.

\subsection{D Sparse Coding}

The aim of this phase is to get the sparse matrices $\left\{\mathbf{X}_{j}\right\}_{j=1}^{N}$ with the given dictionaries $\mathbf{A}$ and $\mathbf{B}$. We re-formulate this problem as

$$
\underset{\mathbf{X}_{j}}{\arg \min }\left\|\mathbf{X}_{j}\right\|_{0} \quad \text { s.t. }\left\|\mathbf{A} \mathbf{X}_{j} \mathbf{B}^{T}-\mathbf{S}_{j}\right\|_{F}^{2} \leqslant \epsilon, \forall j=1,2, \cdots, N
$$


[19] developed a 2D-OMP for 2D sparse signal recovery. With the advantages of low complexity and good performance, we use this algorithm to solve the problem (3). In 2D-OMP, each atom of dictionary is a matrix, which is the outer product of two columns of $\mathbf{A}$ and $\mathbf{B}$ respectively, i.e.,

$\mathbf{D}_{p, q}=\mathbf{A}_{p} \times \mathbf{B}_{q}^{T}=\left(\begin{array}{c}\mathbf{A}_{1, p} \\ \mathbf{A}_{2, p} \\ \vdots \\ \mathbf{A}_{m, p}\end{array}\right) \times\left(\begin{array}{c}\mathbf{B}_{1, q} \\ \mathbf{B}_{2, q} \\ \vdots \\ \mathbf{B}_{m, q}\end{array}\right)^{T}=\left(\begin{array}{ccc}\mathbf{A}_{1, p} \mathbf{B}_{1, q} & \cdots & \mathbf{A}_{1, p} \mathbf{B}_{m, q} \\ \vdots & & \vdots \\ \mathbf{A}_{m, p} \mathbf{B}_{1, q} & \cdots & \mathbf{A}_{m, p} \mathbf{B}_{m, q}\end{array}\right)$

Here, $\mathbf{A}_{p}$ is the $p^{t h}$ column of the matrix $\mathbf{A} . \mathbf{A}_{i, p}, 1 \leq i \leq m$ is an entry of the matrix $\mathbf{A}$, and so do $\mathbf{B}_{q}$ and $\mathbf{B}_{i, q}$. Then $\mathbf{S}_{j}$ can be represented by the weighted sum of $\mathbf{D}_{p, q}$. Suppose that $\widetilde{\mathbf{S}}_{j}=\sum_{p=1}^{n} \sum_{q=1}^{n} \widetilde{\mathbf{X}}_{p, q, j} \mathbf{D}_{p, q}$ is an approximation of $\mathbf{S}_{j}\left(\widetilde{\mathbf{X}}_{p, q, j}\right.$ is an entry of the 3-dimensional matrix $\widetilde{\mathbf{X}} . p, q$ and $j$ are indexes of the three dimensions respectively), $\mathbf{X}_{j} \in \mathbb{R}^{n \times n}$ can be obtained

$$
\begin{aligned}
\mathbf{X}_{j} & =\underset{\mathbf{X}_{j}}{\arg \min }\left\|\mathbf{S}_{j}-\widetilde{\mathbf{S}}_{j}\right\|_{F}^{2} \\
& =\mathbf{H}^{-1} g
\end{aligned}
$$

where

$$
\mathbf{H}=\left(\begin{array}{ccc}
\operatorname{tr}\left(\mathbf{D}_{p_{1}, q_{1}} \mathbf{D}_{p_{1}, q_{1}}^{T}\right) & \cdots & \operatorname{tr}\left(\mathbf{D}_{p_{1}, q_{1}} \mathbf{D}_{p_{l}, q_{l}}^{T}\right) \\
\vdots & & \vdots \\
\operatorname{tr}\left(\mathbf{D}_{p_{l}, q_{l}} \mathbf{D}_{p_{1}, q_{1}}^{T}\right) & \cdots & \operatorname{tr}\left(\mathbf{D}_{p_{l}, q_{l}} \mathbf{D}_{p_{l}, q_{l}}^{T}\right)
\end{array}\right),
$$

$g=\left[\operatorname{tr}\left(\mathbf{S}_{j}, \mathbf{D}_{p_{1}, q_{1}}^{T}\right), \cdots, \operatorname{tr}\left(\mathbf{S}_{j}, \mathbf{D}_{p_{l}, q_{l}}^{T}\right)\right]^{T} . l$ is the number of the selected atoms in the current iteration. For the details of 2D-OMP, please refer to [19].

\subsection{Dictionary Optimization}

In this phase, we will optimize the low dimensional dictionaries $\mathbf{A}$ and $\mathbf{B}$. Different from [7], we add two log-barrier functions to the objective function to emphasize the full rank and the incoherence constraints, as follows.

$$
\begin{aligned}
h(\mathbf{Q}) & =-\frac{1}{m \log (m)} \log \operatorname{det}\left(\frac{1}{n} \mathbf{Q Q}^{T}\right), \\
r(\mathbf{Q}) & =-\sum_{k<l} \log \left(1-\left(\left(\mathbf{Q}_{k}\right)^{T}\left(\mathbf{Q}_{l}\right)\right)^{2}\right),
\end{aligned}
$$


where $\mathbf{Q} \in \mathbb{R}^{m \times n}$ with $m \leq n$.

Thus, the objective function is formulated as

$$
\begin{aligned}
\underset{\mathbf{A}, \mathbf{B}}{\arg \min } \frac{1}{N} \sum_{j=1}^{N} & \left\|\mathbf{A X}_{j} \mathbf{B}^{T}-\mathbf{S}_{j}\right\|_{F}^{2} \\
& +\lambda[r(\mathbf{A})+r(\mathbf{B})]+\kappa[h(\mathbf{A})+h(\mathbf{B})] .
\end{aligned}
$$

We use the geometric conjugate gradient method on oblique manifold to solve (7). As mentioned in [20], A and B are elements of oblique manifold, and the set $\chi=(\mathbf{A}, \mathbf{B})$ is also an element of oblique manifold. (7) can be formed as

$$
\underset{\chi \in \mathrm{M}}{\arg \min } f(\chi)
$$

where $f(\chi)=\frac{1}{N} \sum_{j=1}^{N}\left\|\mathbf{A} \mathbf{X}_{j} \mathbf{B}^{T}-\mathbf{S}_{j}\right\|_{F}^{2}+\lambda[r(\mathbf{A})+r(\mathbf{B})]+\kappa[h(\mathbf{A})+h(\mathbf{B})]$

For every point $\chi \in \mathcal{M}$ ( $\mathcal{M}$ is a smooth Riemannian sub-manifold of some Euclidean spaces), there is a real space called tangent space $\mathbf{T}_{\chi} \mathcal{M}$, which consisting of all possible directions that tangentially pass through $\chi . \Xi \in \mathbf{T}_{\chi} \mathcal{M}$ is called as a tangent vector. The Riemannian gradient of $f$ at point $\chi$ is an element of the tangent space $\mathbf{T}_{\chi} \mathcal{M}$ that points to the direction of the steepest ascent of the objective function on the manifold. The Riemannian gradient $\mathcal{G}(\chi)$ is the orthogonal projection of the gradient $\nabla f(\chi)$ onto the tangent space $\mathbf{T}_{\chi} \mathcal{M}$. This reads as

$$
\begin{aligned}
\mathcal{G}(\chi) & =\Pi_{\mathbf{T}_{\chi} \mathcal{M}}(\nabla f(\chi)) \\
& =\nabla f(\chi)-\chi \operatorname{ddiag}\left(\chi^{T}(\nabla f(\chi))\right) .
\end{aligned}
$$

Due to the structure of $\chi=(\mathbf{A}, \mathbf{B}), \nabla f(\chi)$ can be read as

$$
\nabla f(\chi)=\left(\frac{\nabla f}{\nabla \mathbf{A}}, \frac{\nabla f}{\nabla \mathbf{B}}\right)
$$

where $\frac{\nabla f}{\nabla \mathbf{A}}$ and $\frac{\nabla f}{\nabla \mathbf{B}}$ are computed by

$$
\begin{aligned}
\frac{\nabla f}{\nabla \mathbf{A}}= & \frac{2}{N} \sum_{j=1}^{N}\left(\left(\mathbf{A X}_{j} \mathbf{B}^{T}-\mathbf{S}_{j}\right)\left(\mathbf{B} \mathbf{X}_{j}^{T}\right)\right) \\
& +\lambda \mathbf{A} \sum_{1 \leq i<j \leq n} \frac{-2 \mathbf{A}_{i}^{T} \mathbf{A}_{j}}{1-\left(\mathbf{A}_{i}^{T} \mathbf{A}_{j}\right)^{2}}\left(e_{i, j}+e_{j, i}\right) \\
& +\frac{-2 \kappa}{n m \log m}\left(\frac{1}{n} \mathbf{A} \mathbf{A}^{T}\right)^{-1} \mathbf{A}
\end{aligned}
$$




$$
\begin{aligned}
\frac{\nabla f}{\nabla \mathbf{B}}= & \frac{2}{N} \sum_{j=1}^{N}\left(\left(\mathbf{A} \mathbf{X}_{j} \mathbf{B}^{T}-\mathbf{S}_{j}\right)^{T}\left(\mathbf{A} \mathbf{X}_{j}\right)\right) \\
& +\lambda \mathbf{B} \sum_{1 \leq i<j \leq n} \frac{-2 \mathbf{B}_{i}^{T} \mathbf{B}_{j}}{1-\left(\mathbf{B}_{i}^{T} \mathbf{B}_{j}\right)^{2}}\left(e_{i, j}+e_{j, i}\right) \\
& +\frac{-2 \kappa}{n m \log m}\left(\frac{1}{n} \mathbf{B B}^{T}\right)^{-1} \mathbf{B},
\end{aligned}
$$

where, $e_{i, j}$ denotes a matrix whose entries are zeros except that the entry of the $i^{t h}$ element in the $j^{\text {th }}$ column is one.

In the conjugate gradient method (CG method), for a given point $\chi^{(i)}$ and a search direction $\mathcal{H}^{(i+1)} \in \mathbf{T}_{\chi^{(i+1)}} \mathcal{M}$, a step size $\alpha^{(i)}$ that leads to sufficient decrease of $f$ is determined by

$$
\alpha^{(i)}=\underset{t \geq 0}{\arg \min } f\left(\Gamma\left(\chi^{(i)}, \mathcal{H}^{(i)}, t\right)\right) .
$$

We calculate this variable by non-monotone line search. $\Gamma(\chi, \mathcal{H}, t)$ is called as the geodesic, which is the smooth shortest curve from $\chi \in \mathcal{M}$ in the direction of $\mathcal{H} \in \mathbf{T}_{\chi} \mathcal{M}$ to another point on $\mathcal{M}$. Once $\alpha^{(i)}$ is determined, the next iteration is computed by

$$
\chi^{(i+1)}=\Gamma\left(\chi^{(i)}, \mathcal{H}^{(i)}, \alpha^{(i)}\right) .
$$

In our approach, $\Gamma\left(\chi^{(i)}, \mathcal{H}^{(i)}, \alpha^{(i)}\right)$ is defined as

$$
\Gamma\left(\chi^{(i)}, \mathcal{H}^{(i)}, \alpha^{(i)}\right)=\left(\Gamma\left(\mathbf{A}, \mathbf{H}_{\mathbf{A}}, \alpha^{(i)}\right), \Gamma\left(\mathbf{B}, \mathbf{H}_{\mathbf{B}}, \alpha^{(i)}\right)\right) .
$$

For $\mathbf{A} \in O B(m, n)$ and $\mathbf{H}_{\mathbf{A}} \in \mathbf{T}_{\mathbf{A}} \mathcal{M}, \Gamma\left(\mathbf{A}, \mathbf{H}_{\mathbf{A}}, \alpha^{(i)}\right)$ can be seen as a combination of circles $r(d, h, t)$.

$$
\begin{gathered}
\Gamma(\mathbf{A}, \mathbf{H}, t)=\left[r\left(\mathbf{A}_{1}, \mathbf{H}_{1}, t\right), r\left(\mathbf{A}_{2}, \mathbf{H}_{2}, t\right), \cdots, r\left(\mathbf{A}_{n}, \mathbf{H}_{n}, t\right)\right] . \\
r(d, h, t)=\left\{\begin{array}{cc}
d & \text { if }\left\|h_{2}\right\|=0 \\
d \cos \left(t\|h\|_{2}\right)+h \frac{\sin \left(t\|h\|_{2}\right)}{\|h\|_{2}} & \text { otherwise }
\end{array}\right.
\end{gathered}
$$

Also, $\Gamma\left(\mathbf{B}, \mathbf{H}_{\mathbf{B}}, \alpha^{(i)}\right)$ can be obtained similarly.

Except the step size $\alpha^{(i)}$, the search direction $\mathcal{H}^{(i+1)} \in \mathbf{T}_{\chi^{(i+1)}} \mathcal{M}$ is also needed to be determined. $\mathcal{H}^{(i+1)}$ is a linear combination of the gradient $\mathcal{G}^{(i+1)}$ and the previous search direction $\mathcal{H}^{(i)}$ in the CG method. However, $\mathcal{H}^{(i)} \in \mathbf{T}_{\chi^{(i)}} \mathcal{M}$ and $\mathcal{G}^{(i+1)} \in \mathbf{T}_{\chi^{(i+1)}} \mathcal{M}$ belong to different tangent spaces, the addition operator 
between two different tangent spaces is not defined. Thus, we need to map $\mathcal{H}^{(i)}$ from $\mathbf{T}_{\chi^{(i)}} \mathcal{M}$ to $\mathbf{T}_{\chi^{(i+1)}} \mathcal{M}$. The parallel transport $\mathcal{T}\left(\Xi, \chi^{(i)}, \mathcal{H}^{(i)}, \alpha^{(i)}\right)$ is defined to transport a tangent vector $\Xi=\left(\Xi_{\mathbf{A}}, \Xi_{\mathbf{B}}\right) \in \mathbf{T}_{\chi^{(i)}} \mathcal{M}$ along $\Gamma\left(\chi^{(i)}, \mathcal{H}^{(i)}, \alpha^{(i)}\right)$ to the tangent space $\mathbf{T}_{\chi^{(i+1)}} \mathcal{M}$.

$$
\mathcal{T}(\Xi, \chi, \mathcal{H}, t)=\left[\mathcal{T}\left(\Xi_{\mathbf{A}}, \mathbf{A}, \mathbf{H}_{\mathbf{A}}, t\right), \mathcal{T}\left(\Xi_{\mathbf{B}}, \mathbf{B}, \mathbf{H}_{\mathbf{B}}, t\right)\right]
$$

$\mathcal{T}\left(\Xi_{\mathbf{A}}, \mathbf{A}, \mathbf{H}_{\mathbf{A}}, t\right)$ can be seen as a combination of a transportation of a tangent vector $\xi$ along the circle $r(d, h, t)$, i.e.,

$$
\begin{gathered}
\mathcal{T}(\Xi, \mathbf{A}, \mathbf{H}, t)=\left[\mathcal{T}\left(\Xi_{1}, \mathbf{A}_{1}, \mathbf{H}_{1}, t\right), \cdots, \mathcal{T}\left(\Xi_{n}, \mathbf{A}_{n}, \mathbf{H}_{n}, t\right)\right] \\
\mathcal{T}(\xi, d, h, t)=\xi-\frac{\xi^{T} h}{\|h\|_{2}}\left(d\|h\|_{2} \sin \left(t\|h\|_{2}\right)\right. \\
\left.+h\left(1-\cos \left(t\|h\|_{2}\right)\right)\right)
\end{gathered}
$$

Similar to $\mathcal{T}\left(\Xi_{\mathbf{A}}, \mathbf{A}, \mathbf{H}_{\mathbf{A}}, \mathbf{t}\right), \mathcal{T}\left(\Xi_{\mathbf{B}}, \mathbf{B}, \mathbf{H}_{\mathbf{B}}, \mathbf{t}\right)$ can be obtained. So the new search direction is computed by

$$
\mathcal{H}^{(i+1)}=-\mathcal{G}^{(i+1)}+\beta \mathcal{T}\left(\mathcal{H}^{(i)}, \chi^{(i)}, \mathcal{H}^{(i)}, \alpha^{(i)}\right) .
$$

We summarize our proposed ASeDiL in Algorithm 1.

\section{Experimental Results}

In this section, we compare the results of our algorithm with other algorithms, such as SeDiL proposed in [7], K-SVD proposed in [10].

In the experiments, we choose 10000 patches of size $8 \times 8$ from different uncorrupted images. The training patches are normalized with zero mean and unit $\ell_{2}$ norm. For the dictionaries $\mathbf{A}$ and $\mathbf{B}$, we initialized them by the MATLAB function randn with normalized columns. Then the initial dictionary $\mathbf{D}$ (denoted as InitD) can be obtained through the Kronecker product of $\mathbf{A}$ and $\mathbf{B}$. Both the sizes of $\mathbf{A}$ and $\mathbf{B}$ are $8 \times 16$ in SeDiL and ASeDiL, and the size of InitD in KSVD is $64 \times 256$. Then we optimize the dictionaries by ASeDiL, SeDiL [7] and K-SVD [10]. In the process of dictionary optimization, we set $\lambda=10^{-3}$ and $\kappa=10^{-3}$ in our algorithm. In the SeDiL, we set the weighting parameters of

sparsity and mutual coherences to $\frac{0.0135}{N}$ and $\frac{0.0129}{N}$ (where $N$ is the number of the training samples), as it is provided in the source code of [7]. The max_iteration is set as $\mathbf{5 0 0}$ for both SeDiL and ASeDiL. 


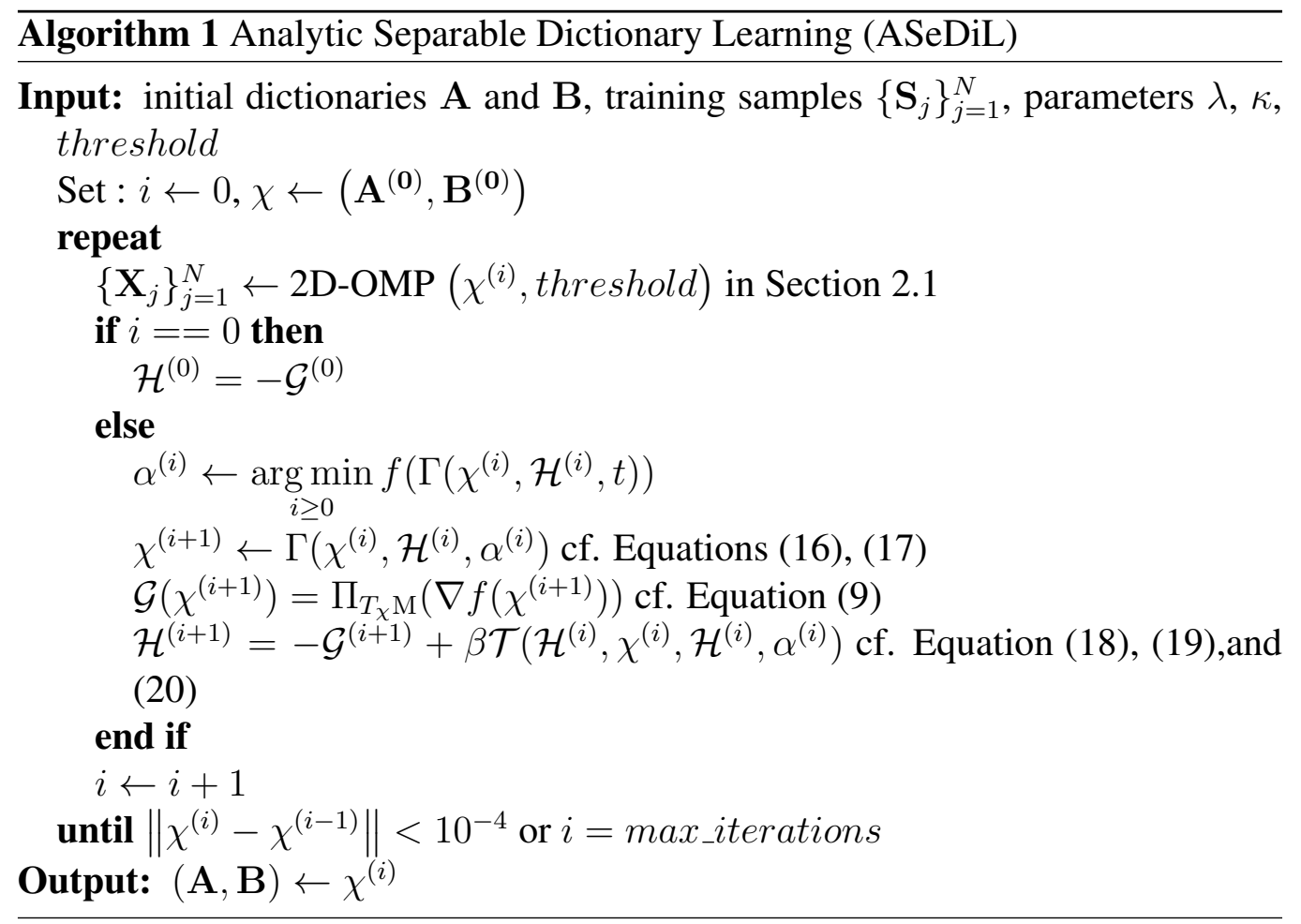

Figure 1 shows the relations between $\frac{1}{N} \sum_{j=1}^{N}\left\|\mathbf{A} \mathbf{X}_{j} \mathbf{B}^{T}-\mathbf{S}_{j}\right\|_{F}^{2}$ and the iteration number. It can be seen that $\frac{1}{N} \sum_{j=1}^{N}\left\|\mathbf{A} \mathbf{X}_{j} \mathbf{B}^{T}-\mathbf{S}_{j}\right\|_{F}^{2}$ decreases much faster in our algorithm than in the SeDiL. The updating procedure stopped after 120 iterations in our algorithm.

At last, the 2D-OMP algorithm is used for sparse representation of the noisy image $\mathbf{y}$ based on the dictionaries obtained by SeDiL, and ASeDiL, as mentioned above. The reconstructions can be expressed as

$$
\underset{\left\{\mathbf{X}_{j}\right\}_{j=1}^{N}}{\arg \min } \frac{1}{N} \sum_{j=1}^{N}\left\|\mathbf{X}_{j}\right\|_{0} \quad \text { s.t. }\left\|\mathbf{A} \mathbf{X}_{j} \mathbf{B}^{T}-\mathbf{y}\right\|_{F}^{2} \leq \varepsilon .
$$

After the sparse representation coefficients are obtained, the reconstructed image can be represented by

$$
\tilde{\mathbf{y}}=\mathbf{A X B}^{T}
$$




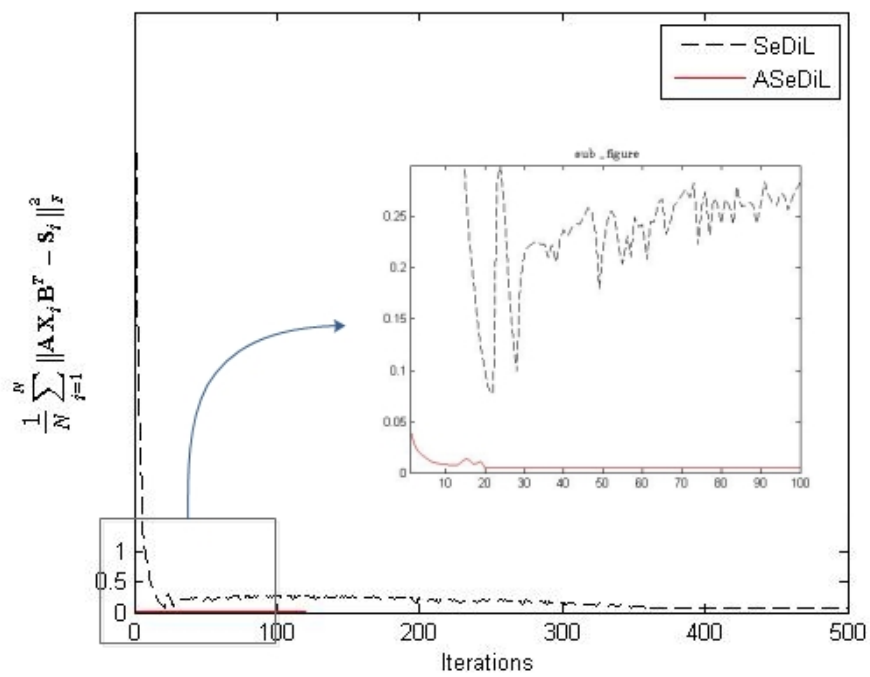

Figure 1: The relations of $\frac{1}{N} \sum_{j=1}^{N}\left\|\mathbf{A} \mathbf{X}_{j} \mathbf{B}^{T}-\mathbf{S}_{j}\right\|_{F}^{2}$ and the number of iterations

In order to show the effectiveness of our algorithm, we also calculate the learning time of ASeDiL by using different algorithms in the first phase. In this experiment, ASeDiL_OMP denotes that OMP is used in the first phase. ASeDiL_2D_OMP denotes that $2 \mathrm{D}-\mathrm{OMP}$ is used to get sparse coding coefficients. Besides the sizes of $\mathbf{A}$ and $\mathbf{B}$, the rest of the parameters are same. The results are shown in Table 1 .

Table 1: Time consumption of each iteration (seconds) in the dictionary training. process.

\begin{tabular}{c|c|c|c} 
The size of A and B & $8 \times 16$ & $8 \times 32$ & $8 \times 64$ \\
\hline ASeDiL_OMP & 23.5742 & 34.0369 & 44.9501 \\
ASeDiL_2D_OMP & $\mathbf{2 1 . 0 0 9 7}$ & $\mathbf{2 6 . 7 8 2 4}$ & $\mathbf{2 9 . 2 0 2 9}$ \\
\hline The size of D & $64 \times 256$ & $64 \times 1024$ & $64 \times 4096$ \\
\hline K-SVD & 26.5011 & 274.8310 & 328.0911
\end{tabular}

\subsection{Image De-noising}

In this section, we present the results of image de-noising based on the dictionaries obtained above. We measure the quality of the reconstructed images by the peak signal to noise ratio (PSNR) and the structure similarity index (SSIM) [21]. Here, the values of SSIM range from 0 to 1 . A big SSIM value means better 
reconstruction result. As shown in Table 2, we present the average PSNR and SSIM (denoted as APSNR and ASSIM) of 5 images: Lena, Barbara, Boat, House and Parrot.

The de-noised results of the Boat image are shown in Figure 2. We also show the relations of the APSNR and ASSIM with the increasing of noise level in Figure 3. It can be seen that our algorithm outperforms SeDiL up to about $1 \mathrm{~dB}$ for APSNR. It is much better than other algorithms for SSIM with the increasing of noise level.

Table 2: Denoising results.

\begin{tabular}{c|c|c|c|c|c}
\multicolumn{2}{c}{$\sigma_{\text {noise }} /$ PSNR } & $5 / 34.14$ & $10 / 28.13$ & $20 / 22.11$ & $30 / 18.57$ \\
\hline \multirow{4}{*}{ APSNR } & InitD & 25.083 & 24.6405 & 23.83 & 23.42 \\
& K-SVD & 34.9648 & 29.2003 & 27.1975 & 24.6688 \\
& SeDiL & 37.214 & 33.456 & 29.78 & 27.747 \\
& ASeDiL & $\mathbf{3 7 . 9 3 6}$ & $\mathbf{3 4 . 2 6}$ & $\mathbf{3 0 . 7 6}$ & $\mathbf{2 8 . 7 1}$ \\
\hline \multirow{5}{*}{ ASSIM } & InitD & 0.7896 & 0.7385 & 0.6819 & 0.6519 \\
& K-SVD & 0.8979 & 0.8257 & 0.7203 & 0.6807 \\
& SeDiL & 0.945 & 0.8949 & 0.8232 & 0.7697 \\
& ASeDiL & $\mathbf{0 . 9 4 9}$ & $\mathbf{0 . 8 9 8 7}$ & $\mathbf{0 . 8 4 1 8}$ & $\mathbf{0 . 7 9 2 1}$
\end{tabular}

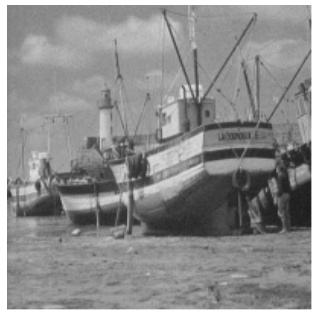

(a) $\sigma_{\text {mast }}=10$

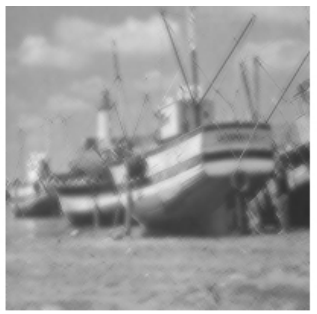

(b) InitD PSRN $=24.6905$

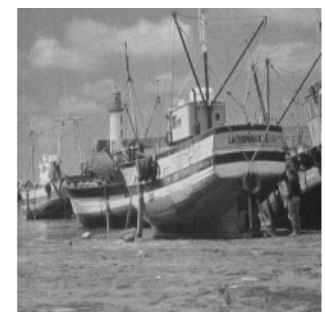

(c) K-SVD PSNR=29.0525

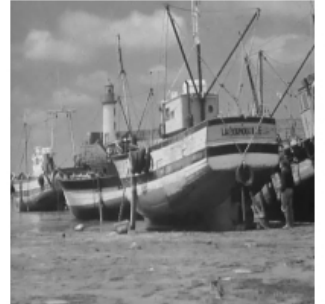

(d) SeDiL PSNR=32.8706

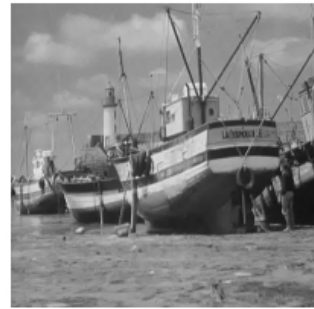

(e) ASeDiL PSNR=33.5174

Figure 2: De-noising results of the Boat image. 

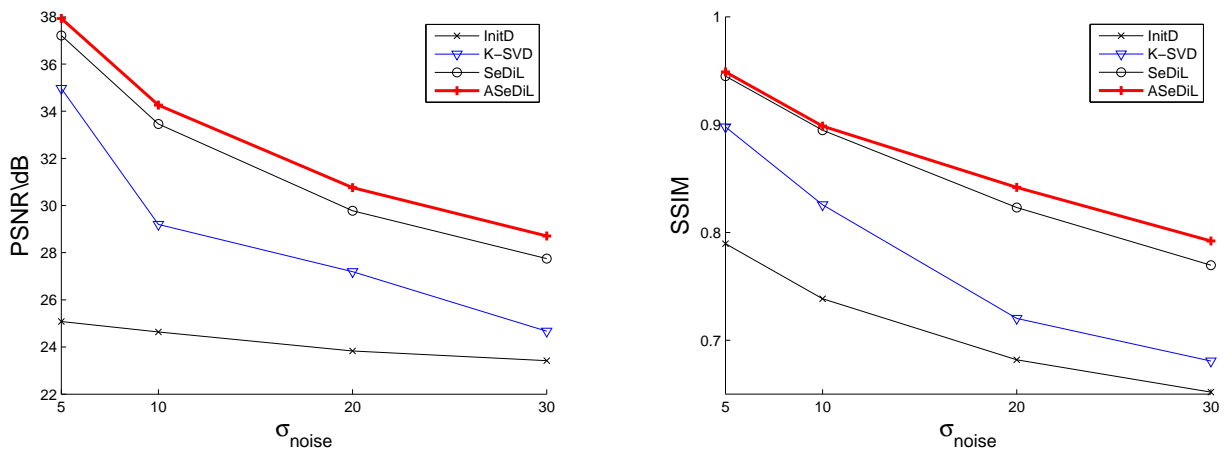

Figure 3: The relations of the APSNR and ASSIM with the increasing of noise level.

\subsection{Face De-noising}

In this experiment, $\mathbf{A}$ and $\mathbf{B}$ are learned from a training set of LFW (labeled faces in the wild crop dataset). There are 13233 frontal face views of different persons of dimension $64 \times 64$. The sizes of $\mathbf{A}$ and $\mathbf{B}$ are $64 \times 96.12000$ images are randomly selected as the training samples, and the remainder images are used as the testing set. The other parameters required for the learning procedure are chosen as that in Section 3.1. Figure 4 shows some of the training images. We also give the APSNR and ASSIM of the test images in Table 3. It can be seen that our algorithm obtains a better performance in preserving the structure of the face images during the images de-noising. Also, some results of face de-noising are presented in Figure 5.

Table 3: Face de-noising results.

\begin{tabular}{c|c|c|c|c}
$\sigma_{\text {noise }}$ & \multicolumn{2}{|c|}{10} & \multicolumn{2}{c}{20} \\
\hline & APSNR & ASSIM & APSNR & ASSIM \\
SeDiL & 29.3072 & 0.8185 & 24.8667 & 0.6349 \\
ASeDiL & $\mathbf{3 0 . 2 0 1 9}$ & $\mathbf{0 . 8 5 7 6}$ & $\mathbf{2 5 . 5 8 2 9}$ & $\mathbf{0 . 7 0 5 4}$ \\
\hline$\sigma_{\text {noise }}$ & \multicolumn{2}{|c|}{30} & \multicolumn{2}{c}{40} \\
\hline SeDiL & 24.5975 & 0.6293 & $\mathbf{2 3 . 9 2 8 8}$ & 0.5780 \\
ASeDiL & $\mathbf{2 4 . 8 6 0 2}$ & $\mathbf{0 . 6 7 6 3}$ & 23.6868 & $\mathbf{0 . 6 1 0 3}$
\end{tabular}

\section{Conclusion}

For the disadvantages of traditional dictionary learning, analytic separable dictionary learning was proposed in this paper. According to the properties of dic- 

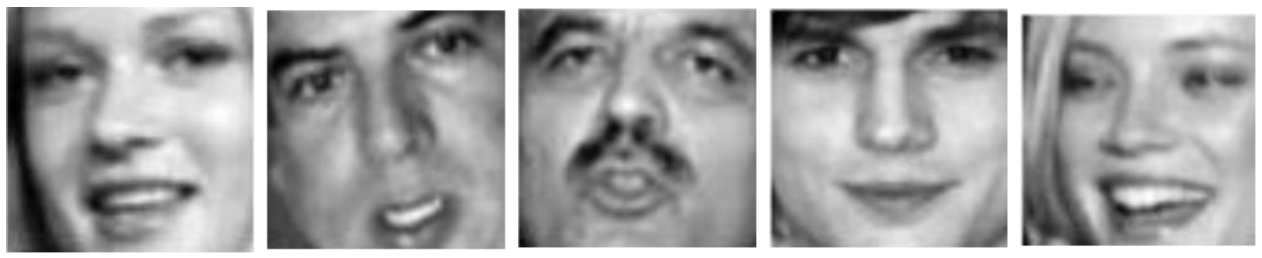

Figure 4: Partial training images.

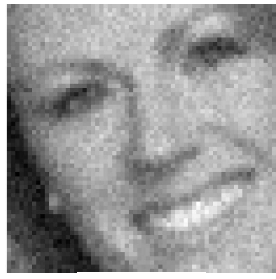

$\sigma_{\text {misut }}=10$

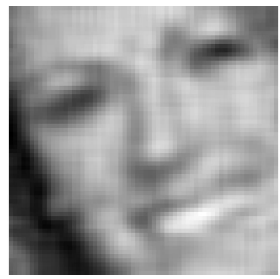

$\mathrm{PSNR}=29.9008$

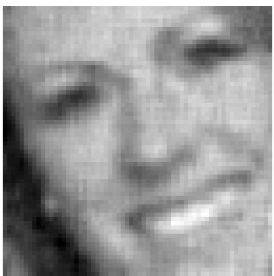

$\mathrm{PSNR}=30.7173$

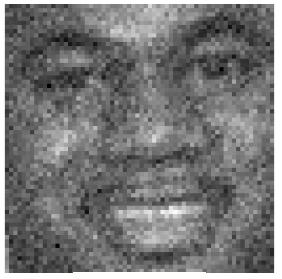

$\sigma_{\text {moist }}=20$

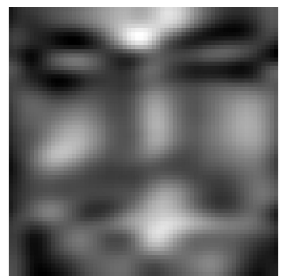

$\mathrm{PSNR}=23.965$

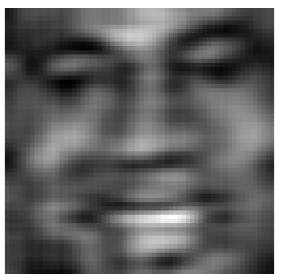

$\mathrm{PSNR}=25.3207$

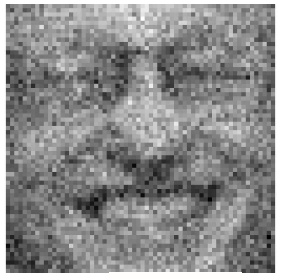

$\sigma_{\text {noist }}=30$

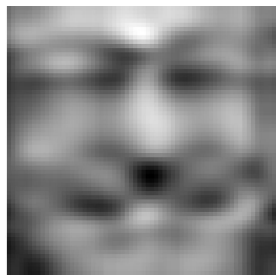

$\mathrm{PSNR}=23.1191$

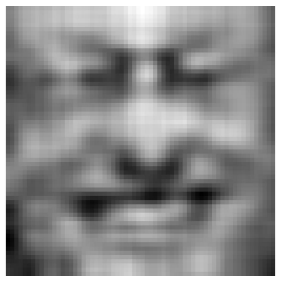

$\mathrm{PSNR}=24.3115$

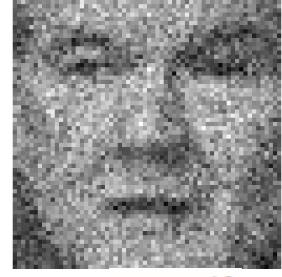

$\sigma_{\text {moust }}=40$

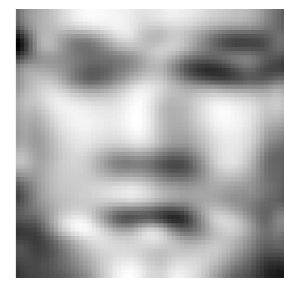

$\mathrm{PSNR}=24.1383$

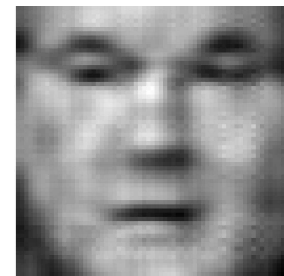

$\mathrm{PSNR}=24.3672$

Figure 5: Face de-noising results. The images of the first row are the corrupted face images, the images in the second row are the de-noising results of SeDiL. The results of ASeDiL are in the third row.

tionary, the rank constraints were introduced into the optimization model. Also, 2D-OMP was used for sparse representation in the dictionary learning process. Compared to the existing algorithm of separable dictionary learning method, our method gained competitive results. 


\section{References}

[1] Z. Lu, L. Zhang, Face recognition algorithm based on discriminative dictionary learning and sparse representation, Neurocomputing 174 (2016) 749755.

[2] M. Elad, M. Aharon, Image denoising via sparse and redundant representation over learned dictionaries, IEEE Transactions on Image Processing 15 (2006) 3736-3745. doi:10.1109/TIP.2006.881969.

[3] S. Yang, Z. Liu, M. Wang, L. Jiao, Multitask dictionary learning and sparse representation based on single-image super-resolution reconstruction, Neurocomputing 74 (2011) 3193-3203.

[4] N. Duo, R. Zhao, Y. Cen, S. Hu, Y. Zhang, Noisy image superresolution reconstruction based on sparse representation, Journal of Computer Research and Development (2015) 943-951.doi:10.7544/issn10001239.2015.20140047.

[5] S. Yang, R. Zhao, Image super-resolution reconstruction based on low-rank and dictionary learning, Journal of Computer Research and Development (2016) 884-891.

[6] S. Hawe, M. Kleinsteuber, K. Diepold, Analysis operator learning and its application to image reconstruction, IEEE Transactions on Image Processing 22 (2013) 2138-2150. doi:10.1109/TIP.2013.2246175.

[7] S. Hawe, M. Seibert, M. Kleinsteuber, Separable dictionary learning, IEEE, Portland, OR, 2013, pp. 438-445.

[8] M. Sadeghi, M. Babaie-Zadeh, C. Jutten, Learning overcomplete dictionaries based on atom-by-atom updating, IEEE Transactions on Image Process 62 (2014) 883-891. doi:10.1109/TSP.2013.2295062.

[9] F. Zhang, R. Zhao, Y. Cen, Adaptive sparse recovery based on difference algorithm, Journal of Computer-Aided Design and Computer Graphics 27 (2015) 1047-1052.

[10] M. Aharon, M. Elad, A. Bruckstein, K-svd: An algorithm for designing overcomplete dictionaries for sparse representation, IEEE Transactions on Signal Processing 54 (2006) 4311-4322. doi:10.1109/TSP.2006.881199. 
[11] H. Zheng, D. Tao, Discriminative dictionary learning via fisher discirmination k-svd algorithm, Neurocomputing 162 (2015) 9-15.

[12] Y. Cen, L. Cen, X. Chen, Z. Miao, Explicit construction of symmetric compactly supported biorthogonal multiwavelets via group transformations, Journal of Computational and Applied Mathematics 244 (2013) 49-66.

[13] X. Zhang, R. Zhao, Y. Cen, Color reconstruction algorithm for grayscale images based on dictionary learning and sparse representation, Journal of Computer-Aided Design and Computer Graphics 26 (2014) 327-331.

[14] L. N. Smith, M. Elad, Improving dictionary learning: Multiple dictionary updates and coefficient reuse, IEEE Signal Processing Letters 20 (2013) 7982. doi:10.1109/LSP.2012.2229976.

[15] H. Bai, G. Li, S. Li, Alternating optimization of sensing matrix and sparsifying dictionary for compressed sensing, IEEE Transactions on Signal Processing 63 (2015) 1581-1594.

[16] N. Qi, Y. Shi, X. Sun, Two dimensional synthesis sparse model, IEEE, San Jose, California, 2013.

[17] Q. Zhang, B. Li, Discriminative k-svd for dictionary learning in face recongnition, 2010. doi:10.1109/CVPR.2010.5539989.

[18] M. Seibert, J. Wormann, R. Gribonval, Separable cosparse analysis operator learning, IEEE, Lison.

[19] Y. Fang, J. Wu, B. Huang, 2d sparse signal recovery via 2d orthogonal matching pursuit, Science China Information Sciences 55 (2012) 889-897. doi:10.1007/s11432-012-4551-5.

[20] N. T. Trendafilov, A continuous-time approach to the oblique procrustes problems, Behaviormetrika (1999) 197181doi:http://dx.doi.org/10.2333/bhmk.26.167.

[21] Z. Wang, A. C. Bovik, H. R. Sheikh, E. P. Simoncelli, Image quality assessment: From error visibility to structural similarity, IEEE Transactions on Image Processing 13 (2004) 600-612. doi:10.1109/TIP.2003.819861. 


\section{Acknowledge}

This work is supported by the National High Technology Research and Development Program (863 Program) of China (No.2014AA15202), National Science Foundation of China (No. 61572067, 61272028, 61502024, 6152063), Beijing

Municipal Natural Science Foundation (No.4162050), Natural Science Foundation of Guangdong Province (No.2016A030313708). 